\title{
Assessment and Measurement of Interleukin 6 in Periodontal Ligament Tissues during Orthodontic Tooth Movement
}

\author{
Azhar Mohammed ${ }^{1}$, Saidath $K^{2}$, Atysha Mohindroo ${ }^{3}$, Akhil Shetty $^{4}$, Veena Shetty ${ }^{5}$, Shama Rao ${ }^{6}$, Prajwal Shetty ${ }^{7}$
}

\begin{abstract}
Aim: To assess and measure the levels of Interleukin-6 (IL-6) expression in the periodontal ligament (PDL) during orthodontic treatment at various intervals of the force application.

Materials and methods: A sample of 60 premolar teeth were divided into controls and test groups of 30 samples each. Each tooth was assigned a time point for its extraction at 2 hours, 15 th day or the 30th day after application of force. Brackets were bonded to the experimental teeth in the mesiodistal center but at 5 degrees to the long axis of the tooth to apply a force of $200-250 \mathrm{~g}$, which was measured by Correx gauge. Gracey's curette was used to scrape off all the periodontal ligament covering the root surface of the extracted teeth. Cytokine IL-6 was measured by the use of an enzyme-linked immunosorbent assay (ELISA) kit at a wavelength of $450 \mathrm{~nm}$.

Results: There was no difference in the levels of IL- 6 at 2 hours for the test and the control groups. IL- 6 concentration showed the maximum levels when measured on the 15th day and showed a decrease on the 30th day.

Conclusion: This study depicted a pattern followed by IL-6 after orthodontic force application, and is a step further in establishing IL- 6 as a potential biomarker for improving the orthodontic treatment in terms of decreased treatment time and decreased side effects.

Clinical significance: IL- 6 can be used as a potential biomarker for efficient treatment in terms of decreased treatment time intervals and decreased side effects of the orthodontic treatment.
\end{abstract}

Keywords: Biomarker, Enzyme-linked immunosorbent assay, Interleukin-6, Periodontal ligament.

World Journal of Dentistry, (2019): 10.5005/jp-journals-10015-1610

\section{INTRODUCTION}

$\mathrm{O}$ rthodontic tooth movement is a result of the cellular elements and the fluid comprising the periodontal ligament which also plays a vital role in the normal functioning of the periodontal ligament surrounding all the teeth in the oral cavity.

The various changes occurring around the orthodontically moving teeth on the histomorphic and molecular level have intrigued the clinicians since the first literature on it was published in 1904-1905 by Sandstedt, ${ }^{2}$ where he demonstrated, area of tension where bone deposition occurs, and an area of compression where bone resorption occurs.

The force applied on the teeth causes a transient inflammatory response which causes vascular dilation in the capillaries leading to migration of leucocytes to the specific site causing the release of area-specific inflammatory mediators like cytokines, prostaglandins, and growth factors. Bone metabolism is carried out by various cytokines namely IL-1, 2, 3, 6, 8, interferon gamma, TNF-alpha, which are expressed in the periodontium. ${ }^{1}$ For bone resorption, IL-1 beta is demonstrated to be the most potent in the amongst these cytokines. ${ }^{2}$ This interleukin has been shown to strongly induce the formation of IL- 6 which therefore is also considered as one of the key mediators in the bone resorption through its effect on osteoclastogenesis. ${ }^{3,4}$

IL- 6 is increased in the periodontal tissues after the orthodontic force is applied. ${ }^{5,6}$ and it plays a key role in the bone resorption process as has been shown by its direct effect on the bone cells. $3,5,7,8$

Various studies have been performed taking samples from animals, ${ }^{3}$ and humans $s^{6,9,10,11}$ demonstrating various cytokines and their variations at different time periods in the periodontal tissues and also in the gingival crevicular fluid. ${ }^{5,12,13}$

PDL is a direct measure of these cytokine levels, as these markers are present directly in its environment. ${ }^{1}$
1-4,7 Department of Orthodontics and Dentofacial Orthopedics, AB Shetty Memorial Institute of Dental Science, Mangaluru, Karnataka, India

${ }^{5}$ Department of Microbiology (Kshema), Nitte University Center for Stem Cell Research and Regenerative Medicine, Mangaluru, Karnataka, India

${ }^{6}$ Nitte University Center for Stem Cell Research and Regenerative Medicine, Mangaluru, Karnataka, India

Corresponding Author: Akhil Shetty, Department of Orthodontics, AB Shetty Memorial Institute of Dental Science, Mangaluru, Karnataka, India, e-mail: akhil_shetty2000@yahoo.com

How to cite this article: Mohammed A, Saidath K, Mohindroo A, Shetty A, Shetty V, Rao S, Shetty P. Assessment and Measurement of Interleukin 6 in Periodontal Ligament Tissues during Orthodontic Tooth Movement. World J Dent 2019;10(2):88-92.

Source of support: Nil

Conflict of interest: None

This in-depth knowledge of the molecular events will aim towards improving the clinical approaches and formulating a treatment plan targeted toward these molecules which then can be used as diagnostic markers and can become potential targets for any therapeutic intervention, ${ }^{1}$ thus helping us in providing improved treatment for the patients. ${ }^{14}$

The aim of this study was to determine the kinetics of IL- 6 expression in the PDL during orthodontic treatment.

\section{Materials and methods}

The study was conducted at the Department of Orthodontics and Dentofacial Orthopedics, AB Shetty Memorial Institute of Dental

(O) The Author(s). 2019 Open Access This article is distributed under the terms of the Creative Commons Attribution 4.0 International License (https://creativecommons. org/licenses/by-nc/4.0/), which permits unrestricted use, distribution, and non-commercial reproduction in any medium, provided you give appropriate credit to the original author(s) and the source, provide a link to the Creative Commons license, and indicate if changes were made. The Creative Commons Public Domain Dedication waiver (http://creativecommons.org/publicdomain/zero/1.0/) applies to the data made available in this article, unless otherwise stated. 
Science, Mangaluru and Nitte University Center for Stem Cell Research and Regenerative Medicine (NUCSReM), Mangaluru, Karnataka, India.

A total of 30 patients requiring two premolar extractions were selected. Among the 30 patients, 30 premolar teeth without orthodontic brackets were taken as the control and 30 premolar teeth with orthodontic brackets were taken as a test which was further divided to 10 premolar teeth each for 2 hours, 15th day and 30th day tests.

Healthy patients without any periodontal bone loss as evaluated in their radiographs were included, and informed consent was taken. Patients with any systemic diseases and those taking, anti-inflammatory drugs, hormonal drugs, or systemic antibiotics for at least 6 months prior to the study were excluded.

The teeth from the same arch were then randomly allocated into control or a test group. Each tooth was then assigned a time point for their extraction at 2 hours, 15th day or the 30th day after application of force. Brackets were bonded to the test teeth in the mesiodistal center but at 5 degrees to the long axis of the tooth (Fig. 1). A NiTi wire of 0.014 dimensions was inserted into the bracket, which applied a $200-250 \mathrm{~g}$ of force to the experimental tooth as measured by Correx gauge (Fig. 2).

Both the control and test teeth were extracted according to their respective group and Gracey's curette was then used to scrape

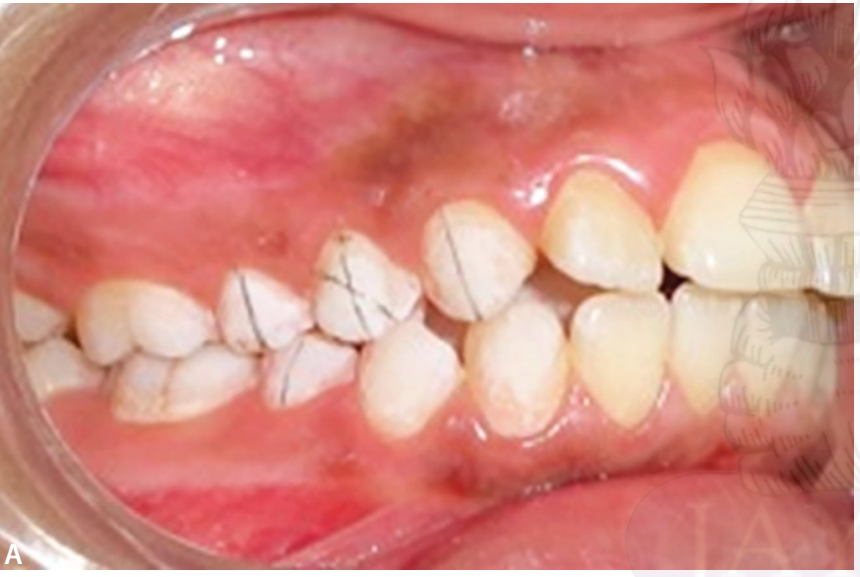

Figs $1 \mathrm{~A}$ and B: Orthodontic bracket was bonded $5^{\circ}$ to the long axis of the tooth

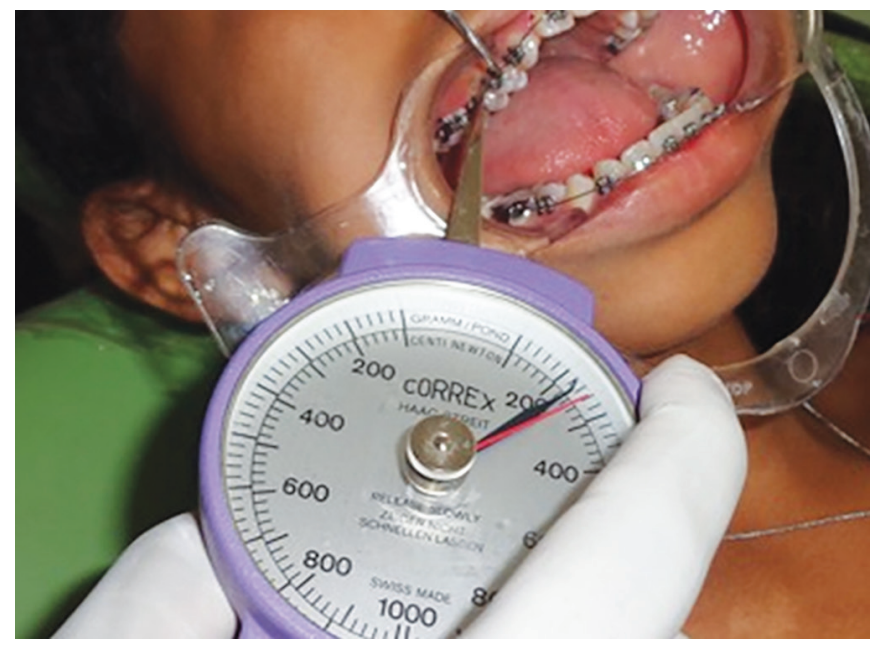

Fig. 2: A force of magnitude 200-250 g, measured by Correx gauge was applied to the premolar off all the periodontal ligament covering the root surface of the extracted teeth (Fig. 3).

The samples were stored for a maximum of 2 months at $-20^{\circ} \mathrm{C}$, and later homogenized with phosphate buffer. This mixture was centrifuged and the supernatant was collected for further analysis using ELISA kit with precoated plates (LEGEND MAX ${ }^{\mathrm{TM}}$, Biolegend) as per the manufacturers' instructions (Biolegend). Cytokine IL-6 was measured as picograms (pg) per $100 \mathrm{mg}$ of tissue at $450 \mathrm{~nm}$ wavelength (Fig. 4).

\section{Results}

Overall IL- 6 concentration was increased and was statistically significant for the test group ( $p<0.05 ; p=0.000$ ) as compared to the control group (Table 1). Man-Whitney U test was done to check the significance for each of the groups (Graph 1).

There was no difference in significance in the values of IL- 6 concentrations for the test and control groups after the extraction of the premolars after 2 hours of force application $(p>0.05$; $p=0.257$ ) (Table 2).

The values of the IL- 6 were statistically significant (Table 3) amongst the test and the control groups at 15 days of force application ( $p<0.05 ; p=0.002$ ).

In the group of 30 days of force application IL- 6 levels were statistically higher in the test group as compared to the control group ( $p<0.05 ; p=0.046$ ) ( Table 4$)$.
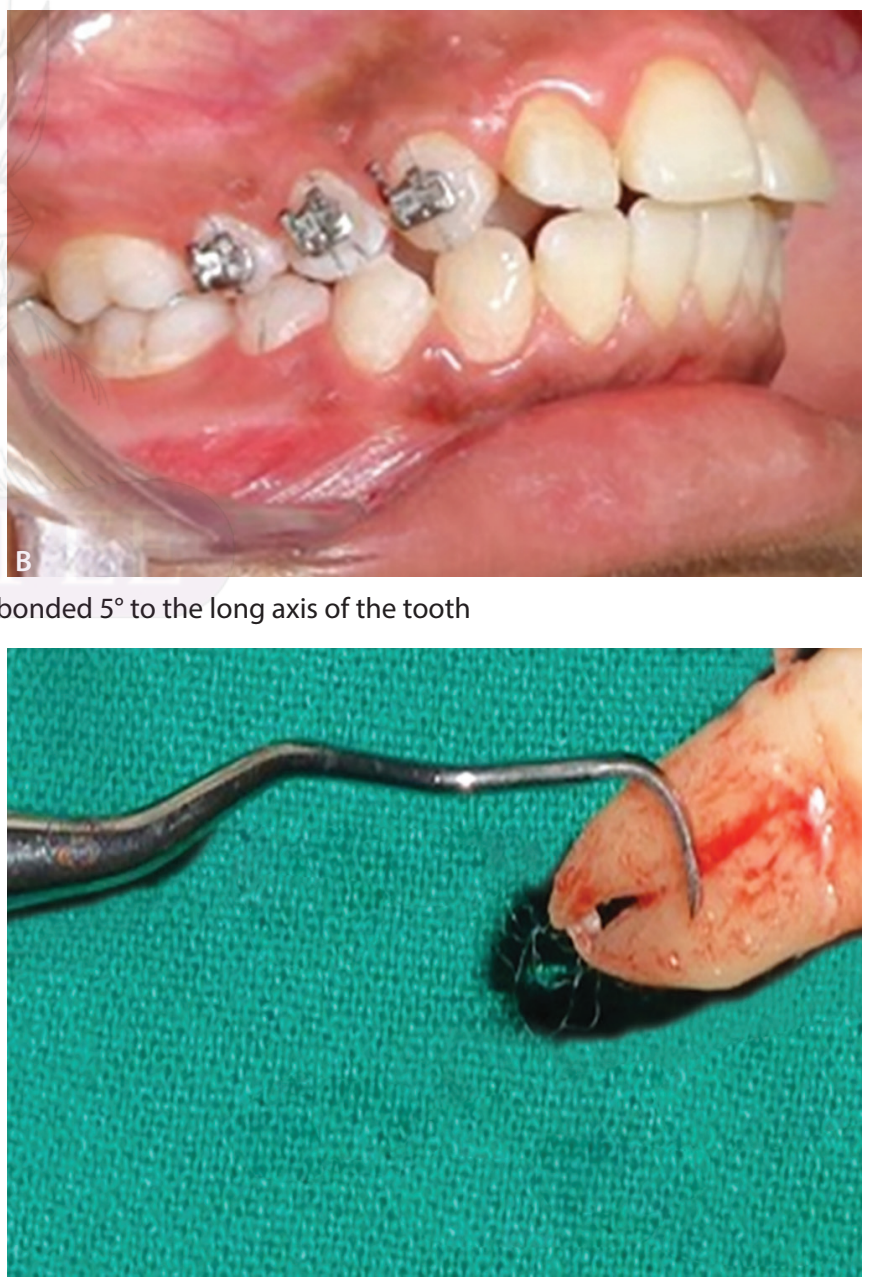

Fig. 3: Gracey's curette used to scrape PDL from the tooth root surface immediately after the tooth was extracted 


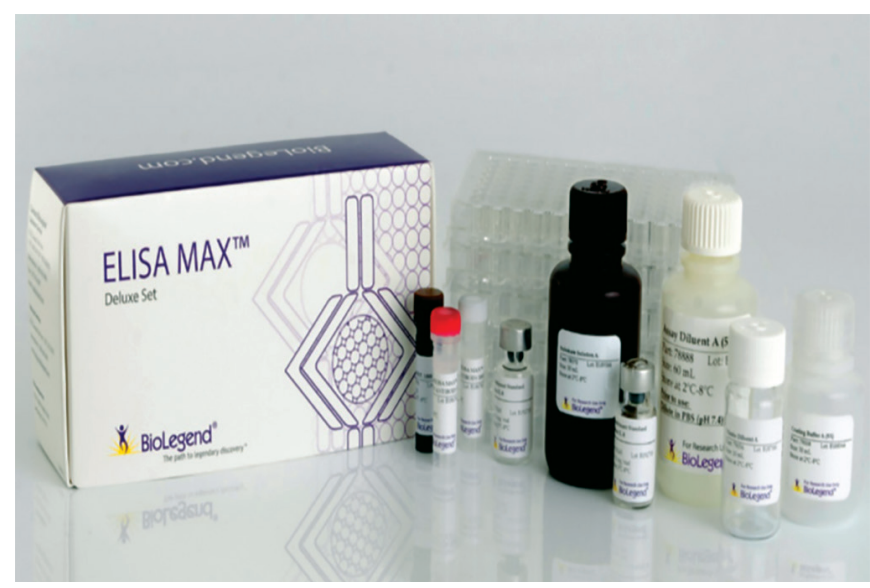

Fig. 4: ELISA kit with precoated plates

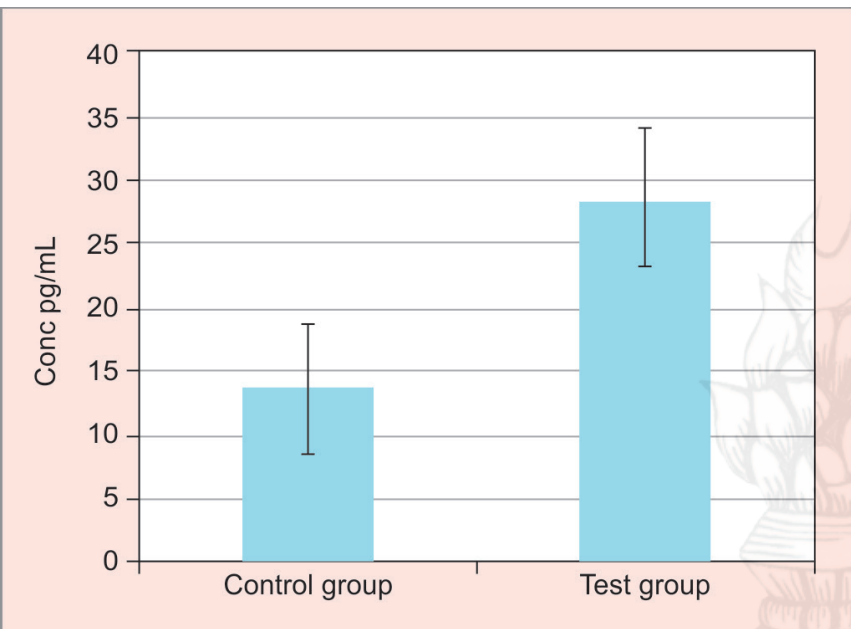

Graph 1: Overall mean ranks for IL-6 concentrations for the test and the control groups

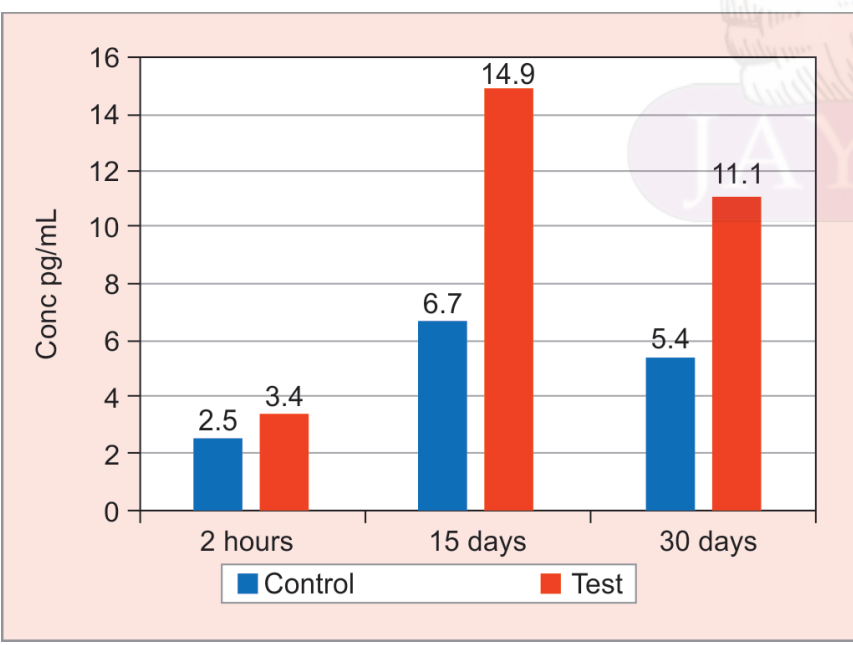

Graph 2: Comparing the mean ranks for the concentration of IL-6 for the force application at 2 hours, 15 days and 30 days

In the group of 30 days of force application IL-6 levels were statistically higher in the test group as compared to the control group ( $p<0.05 ; p=0.046$ ) (Table 4 ), though the levels were less than that measured at the 15th day of force application (Graph 2).
Table 1: Mann-Whitney test (test vs control)

\begin{tabular}{llll}
\hline Group & $N$ & Mean Rank & ${ }^{*}$ p value \\
\hline Control & 30 & 13.69 & 0.000 \\
Test & 30 & 28.60 & \\
\hline Total & 60 & & \\
\hline
\end{tabular}

${ }^{*} p<0.05$ statistically significant, $p>0.05$ Nonsignificant, NS

Table 2: Mann-Whitney test (2 hours of force application)

\begin{tabular}{llll}
\hline Group & $N$ & Mean Rank & ${ }^{*}$ p value \\
\hline Control & 10 & 2.5 & 0.257 \\
Test & 10 & 3.4 & - \\
\hline Total & 20 & - & - \\
\hline
\end{tabular}

${ }^{*} p<0.05$ statistically significant, $p>0.05$ nonsignificant, NS

Table 3: Mann-Whitney test (15 days of force application)

\begin{tabular}{llll}
\hline Group & $N$ & Mean rank & ${ }^{*} p$ value \\
\hline Control & 10 & 6.70 & 0.002 \\
Test & 10 & 14.91 & - \\
\hline Total & 20 & - & - \\
\hline
\end{tabular}

${ }^{*} p<0.05$ statistically significant, $p>0.05$ nonsignificant, NS

Table 4: Mann-Whitney test (30 days of force application)

\begin{tabular}{llll}
\hline Group & $N$ & Mean rank & ${ }^{*}$ p value \\
\hline Control & 10 & 5.40 & 0.046 \\
Test & 10 & 11.1 & - \\
\hline Total & 20 & - & - \\
\hline
\end{tabular}

${ }^{*} p<0.05$ statistically significant, $p>0.05$ nonsignificant, NS

\section{Discussion}

According to Davidson et al. after the application of mechanical stress on the PDL, fluid shifts occur, leading to distortion of the nerve fibers in the PDL, this causes the nerve endings to release neuropeptides, which cause the PDL capillaries to vasodilate, cause extravasation of the plasma, and lead to migration of leukocytes into the PDL extravascular spaces. The released leukocytes produce and release a variety of cytokines which stimulate PDL fibroblasts, alveolar bone cells, and endothelial cells. These inflammatory mediators alter the cellular activity in the periodontal ligament and trigger alveolar bone remodeling. ${ }^{1,2,5,8}$

It has been demonstrated by studies conducted by Reitan and $\mathrm{Rygh}^{2}$ that orthodontic forces leads to activation of osteoblasts depositing new bone in the areas of tension and PDL cells expressed on the compression side lead to the production of enzymes which lead to degradation of the extracellular matrix.

It was stated by Frost in 1963, that in the quest to maintain structural integrity skeleton is continuously being remodeled by a coupled process of resorption and formation of bone in an orchestrated pattern by the cells of osteoclast and osteoblast lineage, which is mediated through locally formed cytokines, 
systemic hormones, growth factors. Similarly, alveolar bone also undergoes this process of bone remodeling, but it is more complicated as there is the presence of the periodontal ligament. The cytokines contribute to the number of pathophysiological mechanisms and are not only specific to inflammatory reactions. ${ }^{11}$ The proinflammatory cytokines include interleukins (ILs 1, 2, 6, 8), interferons, colony stimulating factors, tumor necrosis factors (TNFs), and these have been established to play a key role in the bone remodeling in both the normal physiologic ${ }^{10,11}$ and the force

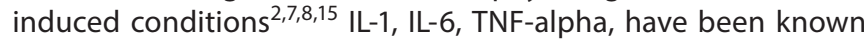
to upregulate the expression of RANKL by the stromal cells and osteoblasts, thus stimulating the bone resorption. ${ }^{7}$

Uematsu et al. stated that IL-6 plays a vital role, especially at the start of the orthodontic tooth movement, helping in the remodeling of the alveolar bone and being a key mediator in the acute inflammation. ${ }^{5,16}$ Also it stimulates the osteoclast formation, therefore helps in bone resorption, all of this is mediated through its autocrine/ paracrine activity. ${ }^{5}$

IL-1, specifically IL-beta is thought to increase the production of IL-6 and thus is its powerful inducer. ${ }^{4,6}$ It is well documented that IL-1 beta increases in response to the mechanical loading of the periodontium, and it is one of the key elements in the acute inflammatory reaction, stimulating the bone resorption. Therefore 1L-1 and IL-6 seem to induce the osteoclastic bone resorption. ${ }^{12}$ Many studies have been done on IL-1 being the prime mediator in the bone resorption process, but very few studies have been carried out on the PDL directly on the changing levels of IL- 6 for different times of force application. Our study will help to establish the changing concentrations of IL- 6 alone in the bone remodeling during orthodontic tooth movement, thus further clarifying its role in orthodontic tooth movement.

In results, our study demonstrated no significant increase in the test group for IL- 6 levels, $(p>0.05)$ when compared with the control group when force is applied for 2 hours. This finding coincided with the study done by Madureira et al. ${ }^{1}$ and Uematsu et al. ${ }^{5}$ who did not find any increased concentrations of the IL- 6 levels in the Pdl and GCF of the extracted teeth at 1 hour and 3 hours of force application, respectively.

Our findings demonstrate IL- 6 concentration reached its peak at 15 days of force application $(p=0.002)$ which is in rhyme with the study by Madureira et al., in which they showed the increased levels of IL-6, in the human PDL at the 3rd day and 12th day. ${ }^{1}$ Study on GCF by Kaya et al. demonstrated the increase of the IL-1 beta, IL-6, TNF- alpha on the day 3 of the force application but it decreased on day 7 and day $10 .{ }^{13}$ Other studies done on the GCF demonstrated IL- 6 increase at 24 hours ${ }^{2,10}$ with no increase during any other time (7th day and 21st day 4, 2nd and 3rd months), ${ }^{10}$ 6 th and 12th months, ${ }^{4,11}$ postorthodontic force application. The results of these studies are not in accordance with the statistically significant result found in our study on the 15th day of the force application. This may be attributed to the low magnitude (10-100 g) of force used in these studies as compared to the magnitude of force used in our study $(200 \mathrm{~g})$ and also due to the measurement in the GCF which cannot be taken as a true reflection of the events occurring in the periodontal tissues. According to Yoo et al., study done on rats the increase in the levels of IL- 6 can be attributed to the fact that after the force has been applied and during the time necrotic tissue is being removed (4th to 20th or 30th day) by the macrophages, more and more leukocytes are being recruited to the site affected due to the force application and also IL- 6 is also known to be produced by other cells like the periodontal fibroblasts, osteoblasts, endothelial cells, bone marrow alveolar cells ${ }^{17}$ which led to the greater increase in the IL- 6 levels at the 15th day of the force application.

IL-6 level in our study decreased at the 30th day of force application as compared to the 15th day $(p=0.046)$ but still was statistically significant. In contradicting studies by Madureira et al. ${ }^{1}$ and Shimizu et al. ${ }^{4}$ on the human periodontal cells, decreased levels of IL- 6 by the 21st day and 21st day, 6 months and 12 months of after force application respectively were reported. This could be attributed to the low magnitude of force (10-100 gm) used in these studies as compared to our study. The value at 30th day (mean rank $=11.1 \mathrm{pg} / \mathrm{mL} ; p=0.046$ ) is however decreased as compared to that at 15 th day (mean rank $=14.9 \mathrm{pg} / \mathrm{mL} ; p=0.002$ ), as the necrotic tissue at this time is almost getting removed thus there is a decrease in the amount of the migrating leucocytes.

Lack of gender and age differentiation is a limitation of the study.

\section{Conclusion}

In the test group, there was no significant rise in the IL- 6 concentration at the 2-hour time point, with the maximum levels when measured on the 15th day and a decrease on the 30th day.

This study depicted a pattern followed by IL- 6 after orthodontic force application, thereby further clarifying the role of IL- 6 in the alveolar bone remodeling during the orthodontic treatment and establishing IL- 6 as a potential biomarker for improving the orthodontic treatment in terms of decreased treatment times, the magnitude of the forces to be used and finally decreasing various potential side effects associated with orthodontic therapy.

\section{Clinical significance}

This study is a step forward in establishing the rhythm followed by IL- 6 during the course of orthodontic treatment when the increased amount of forces are applied, thus using this potential biomarker for the efficient treatment in terms of decreased treatment time intervals and decreased side effects of the orthodontic treatment.

\section{References}

1. Madureira DF, de Albuquerque Taddei S, Abreu $\mathrm{MH}$, et al. Kinetics of interleukin- 6 and chemokine ligands 2 and 3 expression of periodontal tissues during orthodontic tooth movement. American Journal of Orthodontics and Dentofacial Orthopedics 2012;142(4):494-500.

2. Davidovitch Z. Tooth movement. Crit Rev Oral Biol Med 1991; 2:411-450,

3. Alhashimi N, Frithiof L, Brudvik P, et al. Orthodontic tooth movement and de novo synthesis of proinflammatory cytokines. American Journal of Orthodontics and Dentofacial Orthopedics 2001;119(3):307312.

4. Shimizu, N, Ogura N, Yamaguchi M, et al . Stimulation by interleukin-1 of interleukin- 6 production by human periodontal ligament cells. Archives of Oral Biology 1992;37(9):743-748.

5. Uematsu S, Mogi M, Deguchi T. Interleukin(IL)-1beta, IL-6, tumor necrosis factor-alpha, epidermal growth factor, and beta 2-microglobulin levels are elevated in gingival crevicular fluid during human orthodontic tooth movement. J Dent Res 1996;75:562-567.

6. Anastasi G, Cordasco G, Matarese G, et al. An immune histochemical, histological, and electron-microscopic study of the human periodontal ligament during orthodontic treatment. Int J Mol Med 2008;21:545-554.

7. Meikle MC. The tissue, cellular, and molecular regulation of orthodontic tooth movement: 100 years after Carl Sandstedt. Eur J Orthod 2006 Jun;28(3):221-240. 
8. Krishnan V, Davidovitch Z. Cellular, molecular, and tissue level reactions to orthodontic force. Am J Orthod Dentofacial Orthop 2006;129:469. e1-32.

9. Garlet TP, Coelho U, Silva JS, et al. Cytokine expression pattern in compression and tension sides of the periodontal ligament during orthodontic tooth movement in humans. Eur J Oral Sci 2007;115(5):355-362.

10. GarletTP, Coelho U, Repeke CE, et al. Differential expression of osteoblast and osteoclast chemo attractants in compression and tension sides during orthodontic movement. Cytokine 2008;42:330-335.

11. Lee YH, Nahm DS, Jung YK, et al. Differential gene expression of periodontal ligament cells after loading of static compressive force. J Periodontol 2007;78:446-452.

12. Basaran G, Ozer T, Kaya FA, et al. Interleukins 2, 6, and 8 levels in human gingival sulcus during orthodontic treatment. Am J Orthod Dentofacial Orthop. 2006;130:1-6.
13. Ren Y, Vissink A. Cytokines in crevicular fluid and orthodontic tooth movement. Eur J Oral Sci 2008;116(2):89-97.

14. Zainal Ariffin SH, Yamamoto Z, Abidin Z, et al. Cellular and molecular changes in orthodontic tooth movement. The Scientific World Journal 2011;11:1788-803.

15. Davidovitch Z, Nicolay OF, Ngan PW, Shanfeld JL. Neurotransmitters, cytokines, and the control of alveolar bone remodeling in orthodontics. Dent Clin North Am 1988;32(3):411-435.

16. Okada N, Kobayashi M, Mugikura K, et al. Interleukin- 6 production in human fibroblasts derived from periodontal tissues is differentially regulated by cytokines and a glucocorticoid. J Periodontol Res 1997; 32:559-569.

17. Yoo SK, Wartia H, Soma K. Duration of orthodontic force affecting critical response of nitric oxide synthase in rat periodontal ligament. J Med Dent Sci 2004;51:83-88. 